\title{
Power law relationship between diffusion coefficients in multi-component glass forming liquids
}

\author{
Anshul D. S. Parmar ${ }^{1,2}$, Shiladitya Sengupta ${ }^{3}$, Srikanth Sastry ${ }^{1}$ \\ 1 Theoretical Sciences Unit, Jawaharlal Nehru Centre for Advanced Scientific Research, Bengaluru, India. \\ 2 Tata Institute of Fundamental Research, 36/P, Gopanpally Village, \\ Serilingampally Mandal,Ranga Reddy District, Hyderabad, 50010\%, India. \\ 3 Dept. of Fundamental Engineering, Institute of Industrial Science, \\ The University of Tokyo, Komaba 4-6-1, Meguro-ku, Tokyo 153-8505, Japan.
}

\begin{abstract}
The slow down of dynamics in glass forming liquids as the glass transition is approached has been characterised through the Adam-Gibbs relation, which relates relaxation time scales to the configurational entropy. The Adam-Gibbs relation cannot apply simultaneously to all relaxation times scales unless they are coupled, and exhibit closely related temperature dependences. The breakdown of the Stokes-Einstein relation presents an interesting situation to the contrary, and in analysing it, it has recently been shown that the Adam-Gibbs relation applies to diffusion coefficients rather than to viscosity or structural relaxation times related to the decay of density fluctuations. However, for multi-component liquids - the typical cases considered in computer simulations, metallic glass formers, etc. - such a statement raises the question of which diffusion coefficient is described by the Adam-Gibbs relation. All diffusion coefficients can be consistently described by the Adam-Gibbs relation if they bear a power law relationship with each other. Remarkably, we find that for a wide range of glass formers, and for a wide range of temperatures spanning the normal and the slow relaxation regimes, such a relationship holds. We briefly discuss possible rationalisations of the observed behaviour.
\end{abstract}

\section{INTRODUCTION}

An understanding of the dramatic slow down of relaxation dynamics upon approaching the glass transition is central to understanding the behaviour of glass forming liquids. While most theoretical descriptions focus on slow down that is common to various quantities that may be used to describe it, it is well appreciated that the variety of characteristic time scales that one may study, their heterogeneity, etc, display rich and complex variations $1-$ 6. Some commonly studied measures of relaxation are the diffusion coefficient $(D)$, the viscosity $(\eta)$, and the $\alpha$ relaxation time $\left(\tau_{\alpha}\right)$ measured from density correlation functions at a given probe length scale - e.g. the selfintermediate scattering function $F_{s}(k, t)$ at a wave number $k$ corresponding to the peak of the static structure factor. At normal liquid temperatures, the temperature dependence of these quantities are expected to be related, e. g. through the application of the Stokes-Einstein relation. The Stokes-Einstein (SE) relation between the translational diffusion coefficient of a diffusing Brownian particle of mass $m$ and radius $R$ and the shear viscosity of the liquid medium in which it is diffusing:

$$
D=\frac{m k_{B} T}{c \pi R \eta}
$$

where $c$ is a constant determined by the boundary condition (stick or slip) at the surface of the Brownian particle, and $T$ is the temperature of the liquid. This relation is applied to the self diffusion coefficients of liquids and is found to be obeyed quite well 7 However, a hallmark of the glassy dynamics of a liquid approaching the glass transition is the decoupling of different time scales, exemplified by the breakdown of the Stokes-Einstein relation
(SEB) 8 25]. Below a characteristic temperature, the relationship between the diffusion coefficient and the viscosity above does not hold, and instead, it is observed that the diffusion coefficient is related to the viscosity through a fractional power ( i. e., $D \propto \eta^{-\xi}$, with $\xi<1$ ). A similar statement applies to the relationship between the diffusion coefficient and relaxation time scales $\tau(k)$ obtained at different wave vectors $k$, below breakdown temperatures that decrease with a decrease in $k[26$.

The slow down of dynamics is rationalized in terms of a decrease in the configurational entropy $\left(S_{c}\right)$ by the Adam-Gibbs relation [27, which may be written in the form

$$
X=X^{0} \exp \left(\frac{A_{X}}{T S_{c}}\right)
$$

where $X$ is a characteristic relaxation timescale and $A_{X} / S_{c}$ is the corresponding activation energy at a low temperature $T$. With the configurational entropy saturating to a high temperature value, such a form may also further be seen to provide an Arrhenius form at high temperatures, and the Adam-Gibbs parameters may be related to the activation energy in the Arrhenius regime at high temperature and to the fragility of the glass formers 28 31. The Adam-Gibbs relation has been extensively analysed using both experimental and simulation data (see [28, 32, 36] for some examples). In addition to analysing the case of "fragile" liquids which exhibit strongly non-Arrhenius temperature dependence of dynamics, the Adam-Gibbs relation has also been employed to rationalise more complex behaviour of substances like water, which exhibit a fragile-to-strong crossover [37 40]. The ability of the Adam-Gibbs relation to capture dy- 
namical changes in systems with different spatial dimensions has also been tested [41. It is found that 3 and 4 dimensional systems obey the Adam-Gibbs relation well, but 2 dimensional systems exhibit marked deviations, which bear further scrutiny.

The relationship between the configurational entropy and the activation energy for structural relaxation and hence the dynamics is a central idea to various more detailed entropy-based theories of glass transition such as the Random First Order Transition (RFOT) theory 42 44 and the Generalized Entropy Theory [45, 46. Nevertheless, whether this relation should apply primarily to diffusion or structural relaxation as captured by viscosity or the $\alpha$-relaxation time, has not been satisfactorily addressed. This question becomes relevant when the different dynamical quantities decouple, which does indeed occur in glass forming liquids. However, in view of the observed fractional Stokes-Einstein relation, it has been argued that the diffusion coefficient and viscosity, for example, may obey the Adam-Gibbs relation consistently, with different activation energies $A_{X}$ [47 - the fractional exponent simply scales the activation energy in the activated Adam-Gibbs expression. However, such a rationalisation is applicable when one limits attention only to the temperature regime where a single (fractional or inverse) power law relationship holds. If one considers a broad enough temperature range which encompasses the regime in which the diffusion coefficient and viscosity either bear the relationship predicted by the Stokes-Einstein relation, and a regime in which one has the breakdown of the Stokes-Einstein relation, clearly, the Adam-Gibbs relation cannot apply to both diffusion and viscosity simultaneously. Considering such a situation, it was recently shown that indeed, the Adam-Gibbs relation applies to diffusion over the broadest range of temperatures, whereas it is violated for viscosity and relaxation times defined through the self intermediate scattering function, in the presence of the breakdown of the Stokes-Einstein relation 31. The statement that the Adam-Gibbs relation applies primarily to diffusion needs theoretical understanding, but it immediately raises another question, which we address in this manuscript.

Many glass-formers are multi-component substances, with self diffusion coefficients defined for each component. In such a case, the question of which diffusion coefficient(s) the Adam-Gibbs relation applies to arises naturally. This would be a non-issue if the diffusion coefficients bear a constant proportionality, such as what one may expect for Brownian particles from the StokesEinstein relation, but it is observed that this is not the case, and the ratio of diffusion coefficients is temperature dependent [48. Barring constant proportionality, the only other scenario in which the different self diffusion coefficients may consistently obey the Adam-Gibbs relation is that they bear a power law relationship with each other. To test whether such is the case, we have examined self diffusion coefficients in a large range of glass formers, with data obtained both from simulations and experimental measurements. In each case, we have examined data for a wide range of temperatures, from above the 'onset' temperature 4951 of slow dynamics to well below it. Remarkably, we find that in such a wide range of temperatures, a power law relationship is found to hold between the self diffusion coefficients of component species. The fractional exponent is not universal, but varies from values as low as 0.52 to values close to 1. The details of the models and systems we consider are provided in the next section, followed by a discussion.

The rest of the paper is organized as follows: In section II we present the data as well as summarize the details of all the glass-forming systems surveyed here. In section III we discuss the implications of our findings.

\section{DIFFUSION COFFICIENTS FOR MULTI-COMPONENT GLASS FORMING LIQUIDS}

In this section, we describe the details of the glassformers surveyed. The data are shown in Figs. 1, 4 and the relevant parameters are tabulated in Table I. We report the data for diffusion coefficients over a wide range of the temperatures or densities across the onset temperature. The onset temperature has been estimated by considering the deviation from the high temperature Arrhenius behaviour. Vertical lines in the figures mark the diffusion coefficient value of the bigger particles at the onset temperature or the breakdown of the Stokes-Einstein relation. When taken from literature, the source of the data in each case is indicated by appropriate references after the acronym.

\section{A. Simulation}

1. $3 \mathrm{DZrCu}$ 52- Binary mixture of the metallic glass $C u_{33} Z r_{67}$ modelled by embedded atom potential and simulated by NPT molecular dynamics $(M D)$ simulations.

2. $3 \mathrm{DZrNi}$ [53]- 50:50 binary mixture of $Z r_{0.5} N i_{0.5}$ metallic glass simulated by NPT MD.

3. 3DSS- 50:50 binary mixture of soft spheres in 3 dimensions (model details as in 61). We have performed $M D$ simulations in the NVT ensemble have been performed for a temperature range in units of $\frac{\epsilon}{k_{B}}$, where $\epsilon$ is the strength of the potential is $[0.00165,0.00390]$, and the packing fraction is $\phi=0.75$. The packing fraction is defined as $\phi=\left(\sum_{i} \rho_{i} \sigma_{i i}^{3}\right) \pi / 6$, where $i$ represents particle type, $\rho_{i}$ is the number density and $\sigma_{i i}$ the diameter of particle type $i$.

4. 3DLJpoly 54: Polydispersed $L J$ system studied in the NPT ensemble at pressure $P=10 \frac{\epsilon}{\langle\sigma\rangle^{3}}$. 
TABLE I. Power law behavior of diffusivity of different components for various glass forming liquids. "Dim" denotes the spatial dimension, and "R" denotes the radius of a species. Indices $s$ and $f$ denote slow and fast components, respectively. Diffusion coefficient $D^{0}$ corresponds to high-temperature diffusivity. More details about the glass-formers are provided in Sec. II

\begin{tabular}{|c|c|c|c|c|c|c|}
\hline Acronym & Description & Dim & $\begin{array}{c}\text { Exponent } \\
(\gamma)\end{array}$ & $R_{\text {slow }}: R_{\text {fast }} \mid$ & $D_{\text {slow }}^{0}$ & $D_{\text {fast }}^{0}$ \\
\hline $3 \mathrm{DZrCu}[52$ & Embedded atom model for $Z r_{67} C u_{33}$ & 3 & 0.66 & $206: 145$ & $2.45 \mathrm{E}-9$ & $3.57 \mathrm{E}-9$ \\
\hline $3 \mathrm{DZrNi} 53$ & 50:50 binary mixture of $Z r_{0.5} N i_{0.5}$ & 3 & 0.77 & 206:149 & $1.82 \mathrm{E}-11$ & 4.85E-11 \\
\hline $3 \mathrm{DSS}$ & 50:50 mixture of soft spheres & 3 & 0.78 & $1.4: 1$ & $1.02 \mathrm{E}-3$ & $1.41 \mathrm{E}-3$ \\
\hline 3DLJpoly 54 & Polydispersed LJ spheres, NPT MD & 3 & 0.79 & - & $2.45 \mathrm{E}-2$ & $4.71 \mathrm{E}-2$ \\
\hline [26] & 50:50 mixture, square well interaction & 3 & 0.89 & $1.2: 1$ & $9.44 \mathrm{E}-3$ & $1.43 \mathrm{E}-2$ \\
\hline $3 \mathrm{D}$ & 50:50 mixture of hard spheres & 3 & 0.91 & $1.2: 1$ & $5.75 \mathrm{E}-2$ & $7.21 \mathrm{E}-2$ \\
\hline 56 & BKS model of silica & 3 & 0.94 & $1.08: 1$ & $1.35 \mathrm{E}-4$ & $1.68 \mathrm{E}-4$ \\
\hline $\mathrm{K}[56]$ & CHIK model of silica & 3 & 0.94 & $1.08: 1$ & $1.06 \mathrm{E}-4$ & $1.35 \mathrm{E}-4$ \\
\hline 3D-Vitreloy 457 & Bulk metallic glass (Fe:Be) & 3 & 0.52 & $1.56: 1.12$ & $1.70 \mathrm{E}-18$ & $3.44 \mathrm{E}-18$ \\
\hline eloy 4 & ss (Fe:B) & 3 & 0.82 & $1.56: 0.87$ & $5.88 \mathrm{E}-18$ & $1.20 \mathrm{E}-17$ \\
\hline $3 \mathrm{D}-$ & ss (Fe:Co) & 3 & 0.94 & $1.56: 1.52$ & $3 \mathrm{E}-18$ & $1.07 \mathrm{E}-17$ \\
\hline 3D-Vit & $\mathrm{SS}(\mathrm{Fe} \cdot \mathrm{Be})$ & 3 & 0.59 & $1.56: 1.12$ & $1.85 \mathrm{E}-20$ & $1.46 \mathrm{E}-19$ \\
\hline 3D- & glass (Fe:B) & 3 & 0.88 & $1.56: 0.87$ & $6.29 \mathrm{E}-19$ & $2.34 \mathrm{E}-18$ \\
\hline 3D-D1 58 & Bulk metallic glass (Fe:B) & 3 & 0.88 & $1.56: 0.87$ & $3.23 \mathrm{E}-19$ & 7.05E-19 \\
\hline $3 \mathrm{D}-$ & Bulk metallic glass (Fe:B) & 3 & 0.93 & $1.56: 0.87$ & $2.08 \mathrm{E}-19$ & $1.65 \mathrm{E}-18$ \\
\hline 3DLJsoft 29] & 80:20 mixture of modified LJ $(12,11) \mathrm{p}$ & 3 & 0.87 & $1: 0.88$ & $3.24 \mathrm{E}-2$ & $5.44 \mathrm{E}-2$ \\
\hline 3DKA $29,[59$ & $80: 20$ mixt & 3 & 0.91 & $1: 0.88$ & $2.03 \mathrm{E}-1$ & $2.90 \mathrm{E}-1$ \\
\hline & $80: 2$ & 3 & 0.96 & & $\mathrm{E}-2$ & $7.76 \mathrm{E}-2$ \\
\hline 2DKA 47 & interaction & 2 & 0.91 & $1: 0.88$ & $9.01 \mathrm{E}-3$ & $1.18 \mathrm{E}-2$ \\
\hline 2DR12 60] & 50:50 mixture, repulsive $r^{-12}$ power law interaction & 2 & 0.96 & $1.4: 1$ & $7.35 \mathrm{E}-2$ & $7.89 \mathrm{E}-2$ \\
\hline 2DR10 47. & 50:50 mixture, repulsive, $r^{-10}$ power law interaction & 2 & 0.96 & $1.4: 1$ & $6.54 \mathrm{E}-2$ & $7.64 \mathrm{E}-2$ \\
\hline 2DMKA 47 & 65:35 mixture, Kob-Andersen interaction & 2 & 0.99 & $1: 0.88$ & $2.26 \mathrm{E}-2$ & $2.31 \mathrm{E}-2$ \\
\hline 4DKA 47 . & 80:20 mixture of Kob-Andersen interaction & 4 & 0.80 & $1: 0.88$ & $3.65 \mathrm{E}-2$ & 7.96E-2 \\
\hline
\end{tabular}

5. 3DSqW-50:50 binary mixture of particles interacting via an attractive, square well potential 62 . The temperature range is $[0.31,10] \frac{\epsilon}{k_{B}}$ at constant density $\rho=0.77$, where $\epsilon$ is the strength of the potential $[26$.

6. 3DHS [55]- 50:50 binary mixture of hard spheres. The range of packing fractions $[0.425,0.58]$ studied at constant temperature $T=1$.

7. 3DBKS- BKS model for silica in 3 dimensions 56. The temperature range $[2750,6100] K$ studied at the density $2.37 \mathrm{~g} / \mathrm{cm}^{3}$.

8. 3DCHIK- CHIK model for silica in 3 dimensions 56]. The temperature range $[2440,5200] K$ studied at the density $2.20 \mathrm{~g} / \mathrm{cm}^{3}$.

9. 3DLJsoft- 80:20 binary mixture of particles interacting via modified Lennard-Jones (LJ) models in 3 dimensions. The softness is varied by tuning the exponents of the repulsive $(q)$ and attractive $(p)$ part of the LJ potential [29]. The three models we consider have exponents $(q=12, p=11)$, $(q=12, p=6)$, and $(q=8, p=5)$ respectively. The number density for all models is $1.20 \sigma_{A A}^{-3}$.

10. 3DKA- 80:20 binary mixture of particles interacting via the Kob-Andersen potential [63] in 3 dimensions. The number density is $1.20 \sigma_{A A}^{-3}$, and the temperature range is $[0.46,6.0] \frac{\epsilon_{A A}}{k_{B}}$.
11. 2DKA- 80:20 binary mixture of particles interacting via the standard Kob-Andersen potential in 2 dimensions. The temperature range is $[0.93,2.00] \frac{\epsilon_{A A}}{k_{B}}$ studied at the number density $1.20 \sigma_{A A}^{-2}$.

12. 2DMKA- 65:35 binary mixture of particles interacting via the Kob-Andersen potential in 2 dimensions. The temperature range is $[0.45,1.50] \frac{\epsilon_{A A}}{k_{B}}$, studied at the number density $1.20 \sigma_{A A}^{-3}$.

13. 2DR10- 50:50 binary mixture of particles interacting via purely repulsive inverse power law $r^{-10}$ potential in 2 dimensions 64. The temperature range is $[0.52,2.00]$, studied at the number density $0.85 \sigma_{A A}^{-2}$.

14. 2DR12 60 - 50:50 binary mixture of particles interacting via purely repulsive inverse power law $\left(r^{-12}\right)$ potential in 2 dimensions. The temperature range is $[0.4,4.0]$, studied via NPT MD simulations.

15. 4DKA- 80:20 binary mixture of particles interacting via the standard Kob-Andersen potential in 4 dimensions at the reduced number density $1.6 \sigma_{A A}^{-3}$. The temperature range is $[0.80,2.40] \frac{\epsilon_{A A}}{k_{B}}$ [47]. 

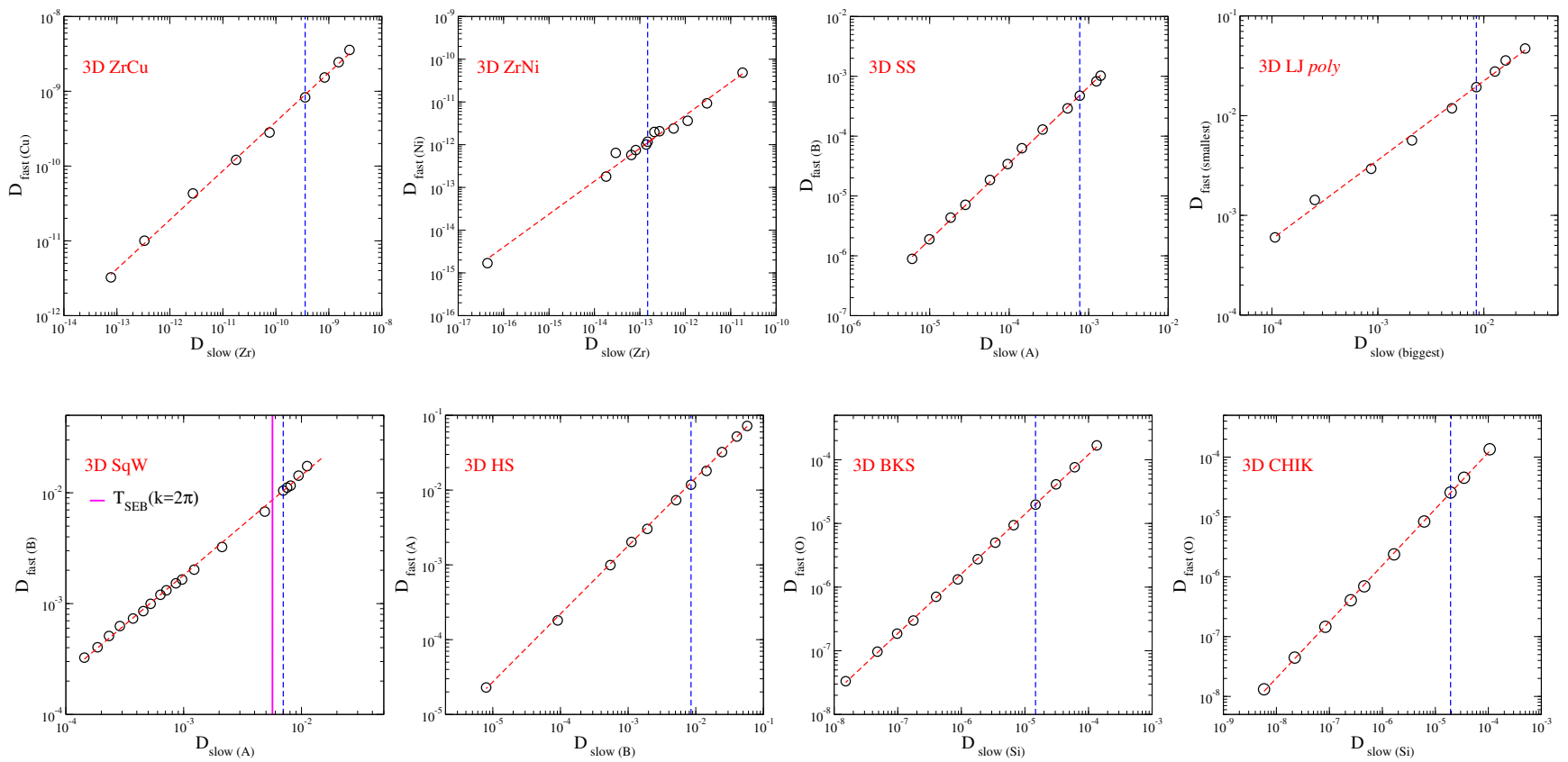

FIG. 1. Power law dependence of diffusion coefficient in several simulation model glass-formers in 3 dimensions. By convention, the slow component is plotted on $\mathrm{X}$ axis and the fast component on Y-axis. Vertical dashed lines indicate the onset temperature/density and continuous lines (for 3DSqW [26]) indicate the Stokes-Einstein breakdown temperature estimated from the structural relaxation times.
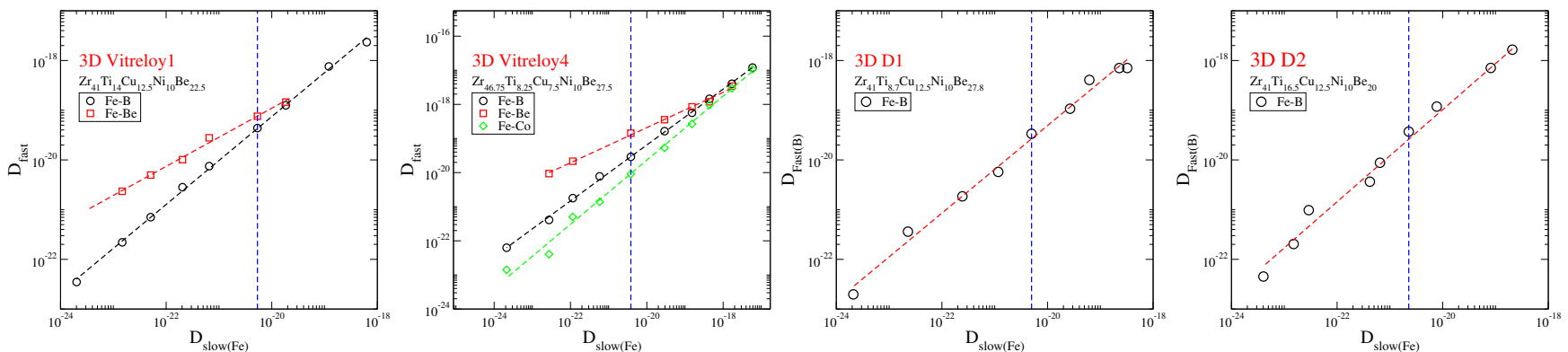

FIG. 2. Power law dependence of diffusion coefficient in several experimental model glass forming liquids. Vertical lines indicate the onset temperature.

\section{B. Experiment}

1. 3D-Vitreloy4 and 3D-Vitreloy1 [57]: Fivecomponent bulk metallic glass alloy Vitreloy4 $\left(Z r_{46.75} \mathrm{Ti}_{8.25} \mathrm{Cu} u_{7.5} \mathrm{Ni} i_{10} B e_{27.5}\right)$ and Vitreloy1 $\left(Z r_{41} \mathrm{Ti}_{14} \mathrm{Cu}_{12.5} \mathrm{Ni}_{10} \mathrm{Be} 22.5\right)$. The data are reported for tracer particles of Boron (B), Iron (Fe), Cobalt (Co) and Beryllium (Be).

2. 3D-D1 and 3D-D2 [58]: They are respectively five-component bulk metallic glass alloys $Z r_{41} T i_{8.7} C u_{12.5} N i_{10} B e_{27.8}$ (D1) and D2 $Z r_{41} T i_{16.5} C u_{12.5} N i_{10} B e_{20}$ (D2). The data are reported for tracer particles of Boron (B) and Iron (Fe).

\section{DISCUSSION}

Figs. 1- 4 show the data of diffusion coefficients where the slow component is plotted along the $\mathrm{X}$-axis, and the fast component is plotted along the $\mathrm{Y}$-axis, by convention. It is clear that in all cases, a fractional power law describes the data well, and very convincingly so in most cases. We also note that the exponent is generally different from 1, with the exponent values being sensitive to the composition and the interaction potential. This is systematically shown in Fig. 3. where softness of the potential and the spatial dimension are varied for a family of glass-formers with Lennard-Jones like interactions. We see that the power law dependence holds in all cases, but the exponent systematically depends on the model details. To emphasize the wide range over which the fractional exponent varies, we also show the scaled plot 

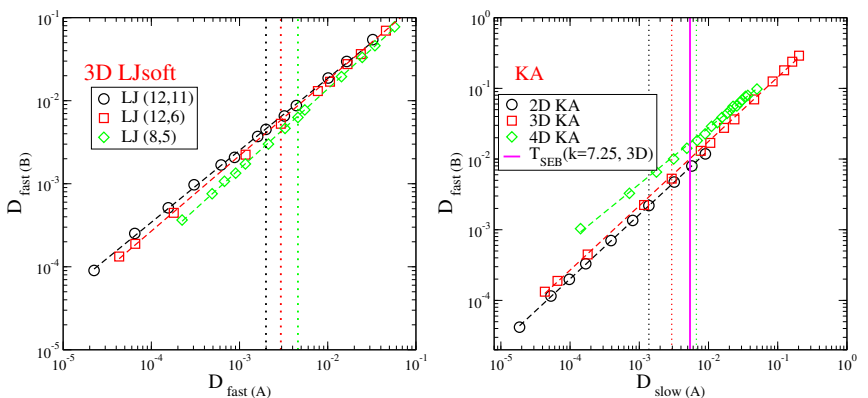

FIG. 3. Examining the power law relationship among diffusion coefficients of different components by systematically varying softness (left panel) and the spatial dimension (right panel) in Lennard-Jones family of model glass-formers. We see that the power law dependence holds in all cases, but the exponent systematically depends on the model details. Vertical dashed lines indicate the onset temperature/density and continuous lines (for 3DKA [26]) indicate the Stokes-Einstein breakdown temperature estimated from the structural relaxation times.

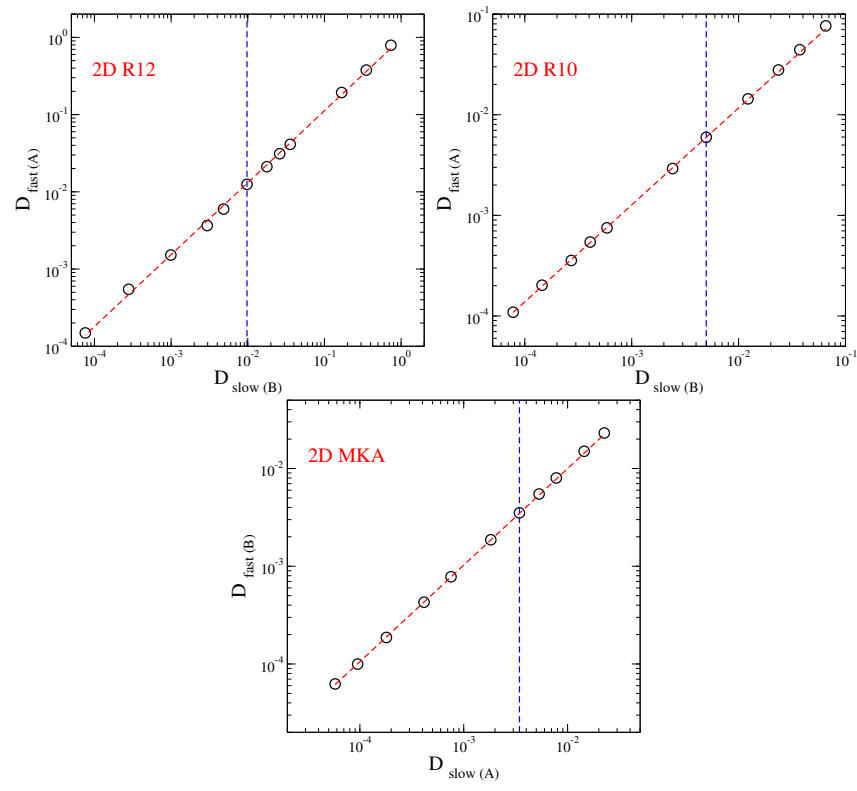

FIG. 4. Power law dependence of diffusion coefficient in several model glass-formers in 2 dimensions. By convention, the slow component is plotted on $\mathrm{X}$ axis and the fast component on Y-axis. Vertical lines indicate the onset temperature.

of diffusion coefficients in Fig. 5. The exponent values and other relevant parameters for different systems are collected in Table I. We also show the temperature $T_{S E B}$ at which the Stokes-Einstein relation breaks down for $3 D K A$ and $3 D S q W$ model glass formers. This highlights that the power law dependence remains valid for a wide dynamical range spanning both the normal SE regime as well as in the supercooled, SEB regime. In this context, we mention that the mode coupling theory (MCT) predicts a power law dependence of the form $D \propto\left(T-T_{c}\right)^{-\gamma}$ for the diffusion coefficient, in a single-component sys-

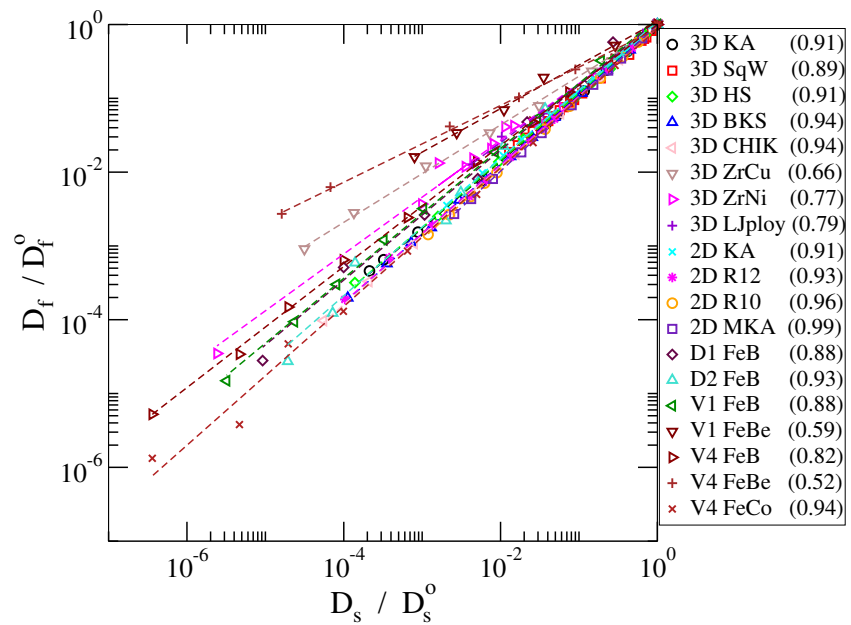

FIG. 5. Scaled plot of diffusion coefficients for all models across dimensions, highlighting the wide range of validity of the power law dependence. Indices $s$ and $f$ denote slow and fast components, respectively. Diffusion coefficients are scaled with the corresponding high-temperature diffusivity $\left(D^{0}\right)$.

tem, where $T_{c}$ is the MCT transition temperature. One may reasonably expect that the same functional form is valid for each component in a multi-component system, thus close to $T_{c}$. Then different components may be related to each other via a power law [65, 66] as recently also verified 67. However, such an argument can be applicable (i) only close to $T_{c}$, and (ii) provided that $T_{c}$ is the same for all components. However, even if the assumption (ii) is valid - which is not obvious a-priori and in fact counter example exists [54] - the MCT argument still can not explain the power law dependence far away from $T_{c}$. In a similar vein, entropy-dependent activation forms for relaxation and scaling relations between diffusion coefficients and appropriate entropy such as the Rosenfeld, the Dzugutov and the Adam-Gibbs relations can not explain the behaviour. However, the observed behaviour is a necessity for these relations to describe the individual component diffusion coefficients consistently. The Rosenfeld and the Dzugutov relations are valid in the normal, high temperature liquid, but break down in the supercooled, low temperature regime. The Adam-Gibbs relation, which motivated the present survey, can not be reliably tested at high temperatures owing to the technical difficulty in measuring configuration entropy. Thus we highlight that the observed power law dependence between diffusion coefficients among different components of a multi-component mixture, should have a wider range of validity that goes beyond the theories of glass transition. Interestingly, however, the seemingly elementary question concerning diffusivity ratios in multi-component liquids has not received theoretical attention despite a vast body of literature studying transport phenomena in varied related contexts. We hope that the present survey will stimulate interest to consider this question more clearly. 


\section{ACKNOWLEDGMENTS}

We thank Dr. Smarjit Karmakar, Indrajit Tah, Rajshekher Das for providing simulation data of 2DKA,
2DMKA and 2DR10 glass formers, Prof. Jürgen Horbach for providing diffusion coefficient data from simulations of BKS and CHIK models and G. Foffi for providing diffusion coefficient data 3DHS model glass former.
[1] L. Berthier and G. Biroli, Reviews of Modern Physics 83, 587 (2011).

[2] L. Berthier, G. Biroli, J.-P. Bouchaud, L. Cipelletti, and W. van Saarloos, Dynamical heterogeneities in glasses, colloids, and granular media, Vol. 150 (OUP Oxford, 2011).

[3] F. W. Starr, J. F. Douglas, and S. Sastry, The Journal of chemical physics 138, 12A541 (2013).

[4] A. Greer, K. Kelton, and S. Srikanth, Fragility of GlassForming Liquids (Hindustan Book Agency, 2013).

[5] S. Karmakar, C. Dasgupta, and S. Sastry, Annu. Rev. Condens. Matter Phys. 5, 255 (2014).

[6] S. Karmakar, C. Dasgupta, and S. Sastry, Reports on Progress in Physics 79, 016601 (2015).

[7] The Stokes' law for the drag force was originally derived for Brownian tracer particles much larger than the particles of the surrounding medium, which was modelled as a continuum fluid obeying macroscopic hydrodynamics. Thus it is quite surprising that this relation actually holds at all for self-diffusion coefficient of the fluid particles, even at high temperatures.

[8] E. Rössler, Physical review letters 65, 1595 (1990).

[9] F. Fujara, B. Geil, H. Sillescu, and G. Fleischer, Zeitschrift für Physik B Condensed Matter 88, 195 (1992).

[10] J. A. Hodgdon and F. H. Stillinger, Physical Review E 48, 207 (1993).

[11] G. Tarjus and D. Kivelson, The Journal of chemical physics 103, 3071 (1995).

[12] M. T. Cicerone and M. D. Ediger, The Journal of chemical physics 104, 7210 (1996).

[13] J. Douglas and D. Leporini, Journal of non-crystalline solids 235, 137 (1998).

[14] H. Sillescu, Journal of Non-Crystalline Solids 243, 81 (1999).

[15] X. Xia and P. G. Wolynes, The Journal of Physical Chemistry B 105, 6570 (2001).

[16] D. Bonn and W. K. Kegel, The Journal of chemical physics 118, 2005 (2003).

[17] L. Berthier, Physical Review E 69, 020201 (2004).

[18] Y. Jung, J. P. Garrahan, and D. Chandler, Physical Review E 69, 061205 (2004).

[19] S.-H. Chong, Physical Review E 78, 041501 (2008).

[20] S.-H. Chong and W. Kob, Physical review letters 102, 025702 (2009).

[21] S. F. Swallen and M. Ediger, Soft Matter 7, 10339 (2011).

[22] J. Langer, arXiv preprint arXiv:1108.2738 (2011).

[23] B. Charbonneau, P. Charbonneau, Y. Jin, G. Parisi, and F. Zamponi, The Journal of chemical physics 139, 164502 (2013).

[24] S. Sengupta and S. Karmakar, The Journal of chemical physics 140, 224505 (2014).

[25] B. P. Bhowmik, R. Das, and S. Karmakar, Journal of Statistical Mechanics: Theory and Experiment 2016, 074003 (2016).
[26] A. D. S. Parmar, S. Shiladitya, and S. Sastry, Physical review letters 119, 056001 (2017).

[27] G. Adam and J. H. Gibbs, The journal of chemical physics 43, 139 (1965).

[28] S. Sastry, Nature 409, 164 (2001).

[29] S. Sengupta, F. Vasconcelos, F. Affouard, and S. Sastry, The Journal of chemical physics 135, 194503 (2011).

[30] A. D. Parmar and S. Sastry, The Journal of Physical Chemistry B 119, 11243 (2015).

[31] A. D. Parmar, P. Kundu, and S. Sastry, Journal of Chemical Sciences 129, 1081 (2017).

[32] C. Angell, Journal of research of the National Institute of Standards and Technology 102, 171 (1997).

[33] A. Scala, F. W. Starr, E. La Nave, F. Sciortino, and H. E. Stanley, Nature 406, 166 (2000).

[34] S. Karmakar, C. Dasgupta, and S. Sastry, Proceedings of the National Academy of Sciences 106, 3675 (2009).

[35] S. Sengupta, T. B. Schrøder, and S. Sastry, The European Physical Journal E 36, 141 (2013).

[36] A. Banerjee, S. Sengupta, S. Sastry, and S. M. Bhattacharyya, Physical review letters 113, 225701 (2014).

[37] K. Ito, C. T. Moynihan, and C. A. Angell, Nature 398, 492 (1999).

[38] S. Sastry, Nature 398, 467 (1999).

[39] F. W. Starr, S. Sastry, E. La Nave, A. Scala, H. E. Stanley, and F. Sciortino, Physical Review E 63, 041201 (2001).

[40] F. W. Starr, C. A. Angell, and H. E. Stanley, Physica A: Statistical Mechanics and its Applications 323, 51 (2003).

[41] S. Sengupta, S. Karmakar, C. Dasgupta, and S. Sastry, Physical review letters 109, 095705 (2012).

[42] T. Kirkpatrick, D. Thirumalai, and P. G. Wolynes, Physical Review A 40, 1045 (1989).

[43] V. Lubchenko and P. G. Wolynes, Annu. Rev. Phys. Chem. 58, 235 (2007).

[44] J.-P. Bouchaud and G. Biroli, The Journal of chemical physics 121, 7347 (2004).

[45] J. Dudowicz, K. F. Freed, and J. F. Douglas, Advances in Chemical Physics 137, 125 (2008).

[46] K. F. Freed, J. Chem. Phys. 141 (2014).

[47] S. Sengupta, S. Karmakar, C. Dasgupta, and S. Sastry, The Journal of Chemical Physics 138, 12 A548 (2013).

[48] H. Schober and H. Peng, Physical Review E 93, 052607 (2016).

[49] S. Sastry, P. G. Debenedetti, and F. H. Stillinger, Nature 393, 554 (1998).

[50] S. Sastry, PhysChemComm 3, 79 (2000).

[51] A. Banerjee, M. K. Nandi, S. Sastry, and S. Maitra Bhattacharyya, The Journal of chemical physics 147, 024504 (2017).

[52] M. Kluge and H. Schober, Physical Review B 70, 224209 (2004).

[53] H. Teichler, Journal of non-crystalline solids 293, 339 (2001). 
[54] R. K. Murarka and B. Bagchi, Physical Review E 67, 051504 (2003).

[55] L. Angelani and G. Foffi, Journal of Physics: Condensed Matter 19, 256207 (2007).

[56] A. Carre, J. Horbach, S. Ispas, and W. Kob, EPL (Europhysics Letters) 82, 17001 (2008).

[57] P. Fielitz, M.-P. Macht, V. Naundorf, and G. Frohberg, Journal of non-crystalline solids 250, 674 (1999).

[58] M.-P. Macht, V. Naundorf, P. Fielitz, J. Rüsing, T. Zumkley, and G. Frohberg, Materials Science and Engineering: A 304, 646 (2001).

[59] S. Sengupta, T. B. Schrøder, and S. Sastry, The European Physical Journal E 36, 141 (2013).

[60] D. N. Perera and P. Harrowell, The Journal of chemical physics 111, 5441 (1999).

[61] L. Berthier and T. A. Witten, Physical Review E 80, 021502 (2009).
[62] I. Saika-Voivod, E. Zaccarelli, F. Sciortino, S. V. Buldyrev, and P. Tartaglia, Physical Review E 70, 041401 (2004).

[63] W. Kob and H. C. Andersen, Physical Review E 51, 4626 (1995).

[64] S. Karmakar, A. Lemaître, E. Lerner, and I. Procaccia, Physical review letters 104, 215502 (2010).

[65] W. Götze, Complex dynamics of glass-forming liquids: A mode-coupling theory, Vol. 143 (OUP Oxford, 2008).

[66] K. Binder and W. Kob, Glassy materials and disordered solids: An introduction to their statistical mechanics (World Scientific, 2011).

[67] N. Bidhoodi and S. P. Das, arXiv preprint arXiv:1803.11483 (2018). 